OPEN ACCESS

Edited by:

Enrico Baruffini,

Università degli Studi di Parma, Italy

Reviewed by:

Theodora Katsila, University of Patras, Greece

Thomy J. L. de Ravel, University Hospitals Leuven, Belgium

*Correspondence: Harvy Velasco hmvelascop@unal.edu.co

Specialty section: This article was submitted to Genetic Disorders, a section of the journal

Frontiers in Genetics

Received: 21 December 2017 Accepted: 28 February 2018 Published: 14 March 2018

Citation: Velasco $H$ and Ramírez-Montaño $D$ (2018) Incidentalome in Neurogenetics: Pathogenic Variant of NSD1 in a Patient With Spinocerebellar Ataxia (SCA).

Front. Genet. 9:86 doi: 10.3389/fgene.2018.00086

\section{Incidentalome in Neurogenetics: Pathogenic Variant of NSD1 in a Patient With Spinocerebellar Ataxia (SCA)}

\author{
Harvy Velasco ${ }^{1 *}$ and Diana Ramírez-Montaño ${ }^{2}$ \\ ${ }^{1}$ Medicina Faculty, Morphology Department, Universidad Nacional de Colombia, Bogotá, Colombia, ${ }^{2}$ Faculty of Health \\ Sciences, Department of Basic Medical Sciences, Universidad Icesi, Cali, Colombia
}

Background: Genetic studies of late-onset sporadic ataxias ( $>40$ years of age) are not routinely indicated. For unresolved cases, next-generation sequencing (NGS) tools, such as whole-exome sequencing (WES), are available for a definitive diagnosis.

Case presentation: Our patient is a woman with a usual facial phenotype and anthropometry, who developed ataxia at 45 years of age, with no relevant family history and an initial clinical approach that ruled out common aetiologies. WES was performed when the patient was 54 years old. The results identified the heterozygous pathogenic variant c.248delA (p.N83MfsX4) in the nuclear receptor-binding SET domain protein 1 (NSD1; MIM 606681) gene (related to Sotos syndrome), which was not associated with ataxia and is not related to the patient's phenotype. Sanger sequencing of NSD1 in two different laboratories confirmed the variant.

Conclusions: NGS findings generally offer valuable information that can be used for clinical decision-making. However, an incidental finding that leads to defining new clinical and bioethical actions is also possible. Consequently, the biological importance of this type of genetic "incidentalome" must be determined.

Keywords: genetic incidentalome, diagnostics test, NSD1, whole-exome sequencing, late-onset sporadic ataxias

\section{BACKGROUND}

Whole-exome sequencing (WES) has changed the genetic analysis of monogenic diseases in the last decade. This diagnostic tool is used to assess both dysmorphological genetic syndromes and several neurological phenotypes, including Mendelian disorders associated with ataxia. WES has a diagnostic efficacy of $>25 \%$; (Baldridge et al., 2017), and it has been helpful to diagnose cases that did not have a definitive diagnosis. It has even facilitated the identification of new syndromes and genes associated with nosological events (Bamshad et al., 2011). In addition to determining a specific diagnosis, WES can also diagnose the coexistence of genetic conditions, which occur in $4.5-7 \%$ cases (Farwell et al., 2015), and it can incidentally identify 
clinically relevant genetic variants unrelated to the patient's phenotype (Green et al., 2017).

There has been a massive increase in incidental genetic findings in studies involving next-generation sequencing (NGS; WES or targeted sequencing) (Green et al., 2017). The clinical term "incidentalome" usually refers to incidental findings in images without clinical suspicion (Kohane et al., 2012). It is also used to describe findings that are not expected in genetic analysis, and genetic incidentalomes are currently estimated to occur in 5-9\% cases (Kohane et al., 2012). However, reports of on such incidentalomes in the literature are few (Vu et al., 2016), and there have been no reports on the underlying neurological pathology.

In recent years, the neurogenetic aetiology of adults has progressed significantly because of the use of NGS technologies (Baldridge et al., 2017). For example, at least 60 types of hereditary ataxia are currently recognized, with an overall prevalence of $15-20$ in 100,000 individuals; the most common forms show autosomal dominant (AD) inheritance (Mancuso et al., 2014).

In cases of suspected hereditary ataxias, the approach can be initiated by exploring clinical elements such as the age of onset, the inheritance mechanism, and the presence of "pure" ataxic symptoms or additional symptoms. Imaging findings should also be analyzed (Klockgether, 2010). Genetic studies may be indicated according to the patient's clinical signs and may include a triplet expansion analysis to study $\mathrm{AD}$ ataxia (Mancuso et al., 2014). However, despite these molecular tools, up to $50 \%$ adult patients with late-onset sporadic ataxias ( $>40$ years of age) are not diagnosed and are classified as late-onset-idiopathic ataxia (Klockgether, 2010).

This paper reports the case of a 54-year-old patient with a history of late-onset idiopathic spinocerebellar ataxia (SCA) in which a definitive diagnosis was not established after biochemical, imaging and genetic studies. WES reported an "incidentalome" in nuclear receptor-binding SET domain protein 1 (NSD1; MIM \#606681) that apparently does not explain the clinical status of the patient.

\section{CASE PRESENTATION}

Our case was a 54-year-old female patient from northern Colombia with a clinical history of 7 years of progressive gait disorder with a slow evolution, frequent falls, vertigo, and dysarthria but without tremor. She did not have a history of alcohol or other toxic substance consumption, trauma or cerebrovascular accidents, or a history of other symptoms or associated chronic pathologies. Family history was negative for parental consanguinity or similar neurological presentation. An ophthalmological examination ruled out retinal and oculomotor involvement.

Abbreviations: ACMG, American College of Medical Genetics and Genomics; $\mathrm{AD}$, autosomal dominant; Ngs, next-generation sequencing; SCA, spinocerebellar ataxia; WES, whole-exome sequencing; HGMD, the human gene mutation database.
The initial MRI at age 47 years showed evidence of cerebellar atrophy as the only relevant finding. The patient underwent several annual follow-up MRI examinations; the last MRI examination at age 53 years did not show any additional findings (Figure 1). Complementary neurological studies such as electromyography and nerve conduction velocities were performed at age 47 years.

At age 54 years, the patient had a height of $161 \mathrm{~cm}$, a weight $67 \mathrm{~kg}$, a body mass index of 24.7 , and a head circumference of $56 \mathrm{~cm}$; her facial appearance and phenotype were normal (Supplementary Figure 1). Her gait was slightly ataxic with a large walking base, greater involvement of toe-to-heel walking, and lateropulsion to the right side. A speech articulation disorder was also noted. Other findings include dysmetria and adiadochokinesia. There was no alteration of deep tendon reflexes or strength, the Romberg and Babinski signs were negative and there was no cranial nerve involvement, nystagmus, or sensory loss.

Complementary blood examination yielded the following results: normal vitamin B12 (309 pg/mL) and vitamin E $(17 \mu \mathrm{g} / \mathrm{mL})$ levels, normal TSH (3.48 mIU/L) levels, and negative HIV and VDRL tests. Other studies performed to rule out normal paraneoplastic syndrome (50 years), including chest $\mathrm{X}$-ray, ultrasonography of the abdomen and mammography, showed no abnormalities.

As an initial genetic study, we performed triplet replication analysis by polymerase chain reaction fragment amplification (at age 49 years) to assess Ataxin1 (SCA 1), Ataxin2 (SCA 2), MJD1 (SCA 3), and CACNA1A (SCA 6). The number of expansions were within the normal range for each gene studied. Karyotype or array-CGH studies were not indicated in the absence of clinical features (major and minor anomalies) that supported chromosomal abnormalities; furthermore, indel-type mutations have not been reported in ataxia-related genes. WES was also performed at age 53 years.

WES was performed on the sequencing coding and flanking intronic regions using the illumine HiSeq2500/4000 system. Illumina CASAVA was used to demultiplex the sequencing reads. The trimmed reads were mapped to the human reference genome (GRCh38). Variants were identified using Samtools and VarScan. Only variants (SNV/small indels) in the coding region and flanking intronic region $( \pm 8 \mathrm{bp})$ with a minor allele frequency (MAF) $<1.5 \%$ were evaluated. Known disease-causing variants (according to HGMD) was evaluated in areas up to $\pm 30 \mathrm{bp}$ of the flanking regions and up to 5\% MAF. MAFs were obtained from the following databases: 1000 genomes, dbSNP, Exome Variant Server, ExAC and an in-house database. The average collage comprised $100 \times$ of at least 30 high-quality sequencing reads per base $(\sim 95 \%$ of the covered target bp $>20 \times)$. This examination revealed a variant c.247delA (p.N83MfsX4) in the NSD1 gene. No other variants were identified by this methodology. This finding was confirmed by Sanger sequencing NSD1 gene (All exons). A likely pathogenic variant was found in the heterozygous state, c.247delA (p.N83MfsX4), which led to a premature stop codon at amino acid 87 (Figure 2). This result was reconfirmed by a European laboratory. Variant 

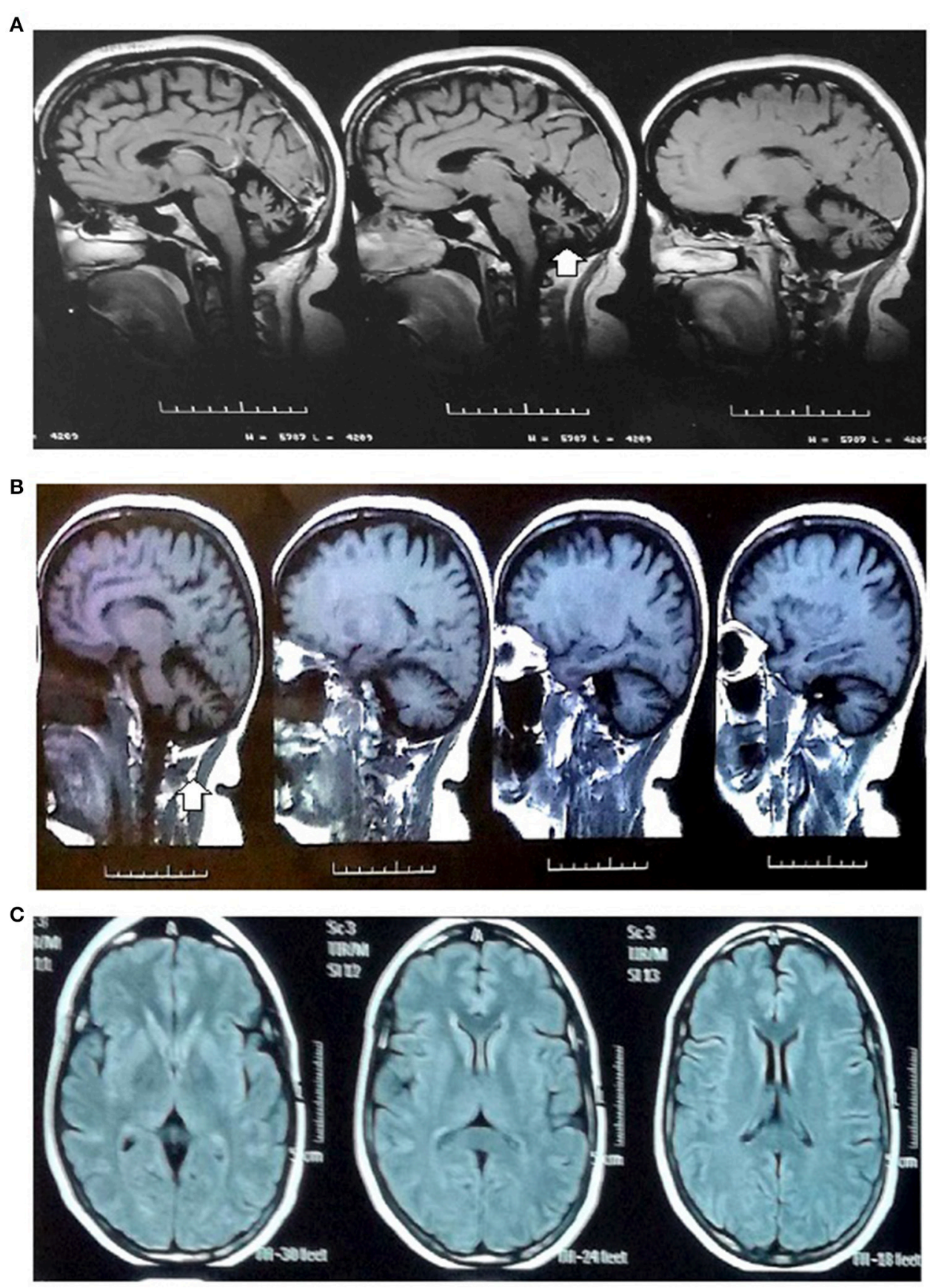

FIGURE 1 | MRI images. MRI images (1.5 tesla): (A,B) Sagittal sections in the T1-weighted MRI images: global cerebellar atrophy with a marked enlargement of the subarachnoid space, thinning of cerebellar folds and vermis (arrow) related to diffuse cerebellar atrophy. (C) Axial section in the T1 sequence: cerebral white matter and ventricles without alterations.

functional prediction software tools Mutation taster, Condel, SIFT, and FATHMM classified it as a Damaging variant (disease causing).

The Ethics Committee of the Universidad Nacional de Colombia (CE-010/2013) approved this study. The patient provided full written consent for publication.

\section{DISCUSSION AND CONCLUSIONS}

A molecular approach to ataxias is indicated when there is suspicion of a monogenic component, although it is not routinely indicated for late-onset sporadic ataxias ( $>40$ years of age) (Klockgether, 2010). The usual form of presentation is the "pure" 


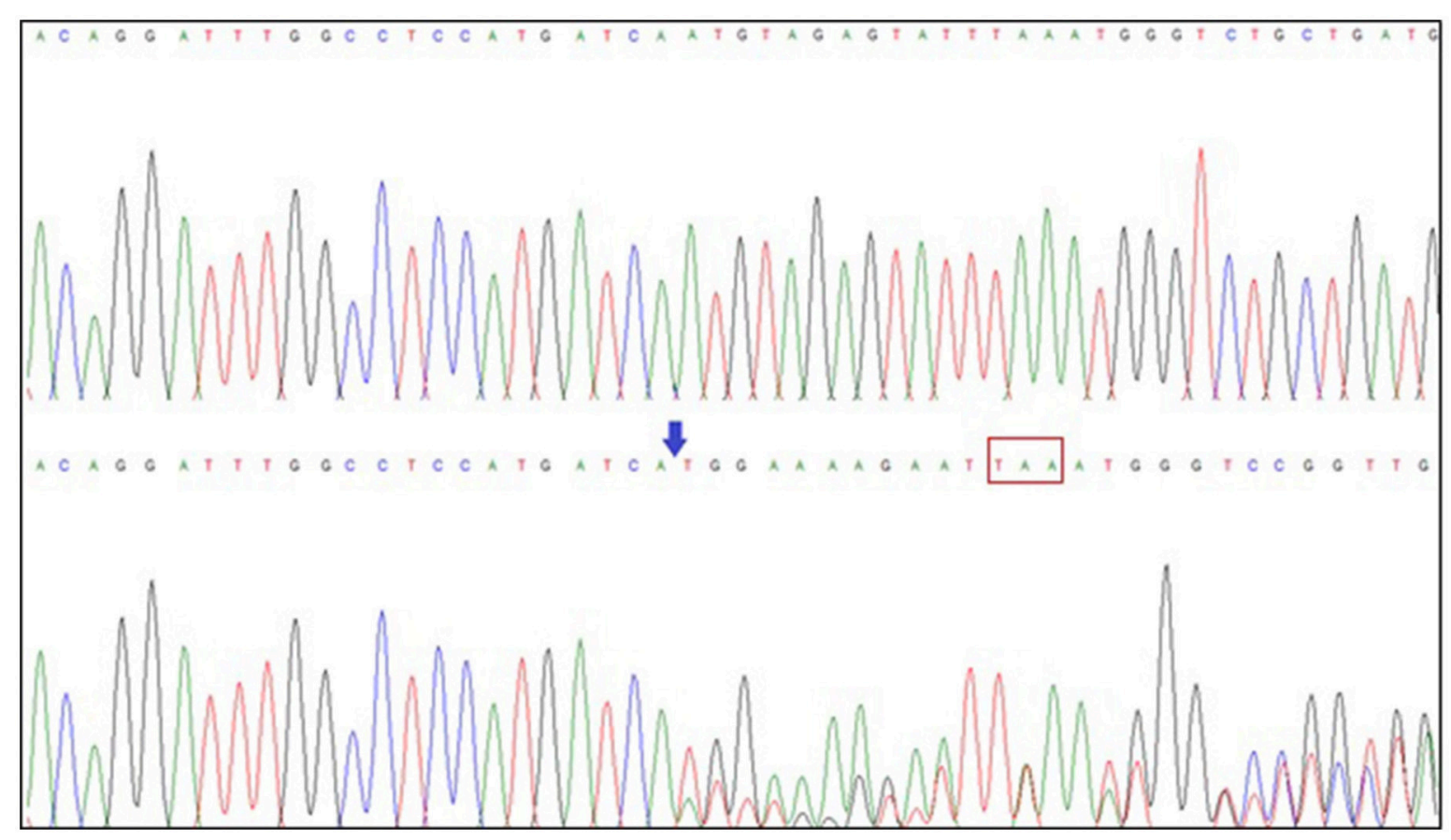

FIGURE 2 | NSD1 gene sequencing. Exon 2 sequence of the NSD1 gene (superior: normal; inferior: patient sequence) showing the deletion of adenine (blue arrow) at position 247 (c.247delA), which has an effect on the protein and generates a premature stop codon at amino acid 87 (red box).

cerebellar form, similar to the case reported here. For this reason, we analyzed the group of SCAs, particularly SCA6 (Klockgether, 2010 ) and types 1,2, and 3 because of their population frequency; the results showed a normal expansion size.

WES can identify causality in diseases with high clinical and genetic heterogeneity, such as ataxia. Its diagnostic performance is close to $40 \%$ (Farwell et al., 2015; Baldridge et al., 2017). In this case, the results did not show the causality; instead, they identified an incidentalome, which generated uncertainty regarding the genetic counseling and subsequent management of the pathology.

In 2014, the American College of Medical Genetics and Genomics (ACMG) (Green et al., 2017) defined 56 actionable genes with informational obligation for incidental findings; NSD1 is not included in this list. The incidental finding of a pathogenic variant in NSD1 by WES in our patient with ataxia raises the possibility of a false-positive finding because this gene is implicated in Sotos syndrome, which is a pathology with a recognizable phenotype (Douglas et al., 2003) not matching the phenotype of the presented case. In addition, there have been no reports of ataxia in patients with Sotos syndrome to date.

The NSD1 gene is located at $5 \mathrm{q} 35.3$ and causes Sotos syndrome in $95 \%$ individuals. Frameshift mutations in this gene are always considered pathogenic (Douglas et al., 2003). The mutation reported here generated a premature stop codon at amino acid 87 that could severely affect the functionality of the protein. In a previous study in which the molecular pathways that are generated from NSD1 interactions in STRING were analyzed, correlation or overlap with pathways involved in the pathogenesis of ataxia was not shown; (Nibbeling et al., 2017) the study showed that the pathways are limited to functions related to possible epigenetic regulatory events.

Kohane et al. (2012) established four elements that must be analyzed to differentiate an incidental finding from a false-positive one: erroneous annotation, sequencing error, incorrect estimation of penetrance, and multiple hypotheses when conducting the test. With regard to the first two elements, the variant found by WES was independently confirmed by two different laboratories through Sanger sequencing, and both laboratories reported the same results. Regarding the incorrect estimation of penetrance, Sotos syndrome displays full penetrance $(100 \%)$ and highly variable expressivity (Douglas et al., 2003). Furthermore, the generational analysis of the patient ruled out the existence of attenuated phenotypes of related syndromes.

In conclusion, the relevance and clinical impact of incidental findings depend on several factors such as the exact definition of the patient's phenotype, the validation and relevance of molecular tests, and the appropriate allocation and classification of the variants (Green et al., 2017). In asymptomatic patients, there is no strong evidence to guide clinical behavior (Kohane et al., 2012). This incidental finding creates an interesting scenario with two possibilities: a new molecular mechanism that allows associating this gene with ataxia or a scenario of incomplete penetrance not previously reported in Sotos syndrome. 


\section{AUTHOR CONTRIBUTIONS}

All authors listed have made a substantial, direct and intellectual contribution to the work, and approved it for publication.

\section{FUNDING}

Authors are employed by and receive a salary from the Universidad Icesi and Universidad Nacional de Colombia respectively.

\section{REFERENCES}

Baldridge, D., Heeley, J., Vineyard, M., Manwaring, L., Toler, T. L., Fassi, E., et al. (2017). The Exome Clinic and the role of medical genetics expertise in the interpretation of exome sequencing results. Genet. Med. 19, 1040-1048. doi: 10.1038/gim.2016.224

Bamshad, M. J., Ng, S. B., Bigham, A. W., Tabor, H. K., Emond, M. J., Nickerson, D. A., et al. (2011). Exome sequencing as a tool for Mendelian disease gene discovery. Nat. Rev. Genet. 12, 745-755. doi: 10.1038/nrg3031

Douglas, J., Hanks, S., Temple, I. K., Davies, S., Murray, A., Upadhyaya, M., et al. (2003). NSD1 mutations are the major cause of Sotos syndrome and occur in some cases of Weaver syndrome but are rare in other overgrowth phenotypes. Am. J. Hum. Genet. 72, 132-143. doi: 10.1086/345647

Farwell, K. D., Shahmirzadi, L., El-Khechen, D., Powis, Z., Chao, E. C., Tippin Davis, B., et al. (2015). Enhanced utility of family centered diagnostic exome sequencing with inheritance model-based analysis: results from 500 unselected families with undiagnosed genetic conditions. Genet. Med. 17, 578-586. doi: 10.1038/gim.2014.154

Green, R. C., Berg, J. S., Grody, W. W., Kalia, S. S., Korf, B. R., Martin, C. L., et al. (2017). Corrigendum: ACMG recommendations for reporting of incidental findings in clinical exome and genome sequencing. Genet. Med. 19:606. doi: 10.1038/gim.2017.18

Klockgether, T. (2010). Sporadic ataxia with adult onset: classification and diagnostic criteria. Lancet Neurol. 9, 94-104. doi: 10.1016/S1474-4422(09)70305-9

\section{ACKNOWLEDGMENTS}

We would like to thank the patient for participation in this study.

\section{SUPPLEMENTARY MATERIAL}

The Supplementary Material for this article can be found online at: https://www.frontiersin.org/articles/10.3389/fgene. 2018.00086/full\#supplementary-material

Supplementary Figure 1 | Facial phenotype. Phenotype no compatible with Sotos syndrome: no macrocrania or overgrowth.

Kohane, I. S., Hsing, M., and Kong, S. W. (2012). Taxonomizing, sizing, and overcoming the incidentalome. Genet. Med. 14, 399-404. doi: 10.1038 /gim.2011.68

Mancuso, M., Orsucci, D., Siciliano, G., and Bonuccelli, U. (2014). The genetics of ataxia: through the labyrinth of the Minotaur, looking for Ariadne's thread. $J$. Neurol. 261, S528-S541. doi: 10.1007/s00415-014-7387-7

Nibbeling, E. A., Delnooz, C. C., de Koning, T. J., Sinke, R. J., Jinnah, H. A., Tijssen, M. A., et al. (2017). Using the shared genetics of dystonia and ataxia to unravel their pathogenesis. Neurosci. Biobehav. Rev. 75, 22-39. doi: 10.1016/j.neubiorev.2017.01.033

Vu, P. Q. Tian, J. J., and Sadun A. A. (2016). Genetic incidentaloma in ophthalmology. JAMA Ophthalmol. 134, 123-124. doi: 10.1001/jamaophthalmol.2015.4679

Conflict of Interest Statement: The authors declare that the research was conducted in the absence of any commercial or financial relationships that could be construed as a potential conflict of interest.

Copyright (c) 2018 Velasco and Ramírez-Montaño. This is an open-access article distributed under the terms of the Creative Commons Attribution License (CC $B Y)$. The use, distribution or reproduction in other forums is permitted, provided the original author(s) and the copyright owner are credited and that the original publication in this journal is cited, in accordance with accepted academic practice. No use, distribution or reproduction is permitted which does not comply with these terms. 